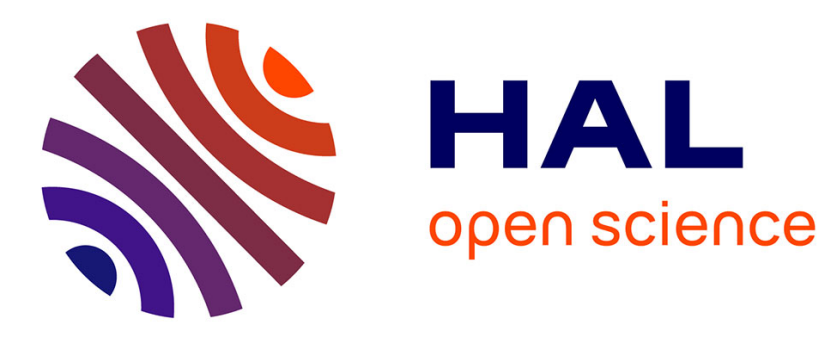

\title{
Hydrophone Array Optimization, Conception, and Validation for Localization of Acoustic Sources in Deep-Sea Mining
}

Valentin Baron, Arthur Finez, Simon Bouley, Florent Fayet, Jerome I. Mars, Barbara Nicolas

\section{To cite this version:}

Valentin Baron, Arthur Finez, Simon Bouley, Florent Fayet, Jerome I. Mars, et al.. Hydrophone Array Optimization, Conception, and Validation for Localization of Acoustic Sources in Deep-Sea Mining. IEEE Journal of Oceanic Engineering, 2021, 46 (2), pp.555 - 563. 10.1109/joe.2020.3004018 . hal03052568

\section{HAL Id: hal-03052568 https://hal.science/hal-03052568}

Submitted on 4 Jan 2021

HAL is a multi-disciplinary open access archive for the deposit and dissemination of scientific research documents, whether they are published or not. The documents may come from teaching and research institutions in France or abroad, or from public or private research centers.
L'archive ouverte pluridisciplinaire HAL, est destinée au dépôt et à la diffusion de documents scientifiques de niveau recherche, publiés ou non, émanant des établissements d'enseignement et de recherche français ou étrangers, des laboratoires publics ou privés. 


\title{
Hydrophone Array Optimization, Conception, and Validation for Localization of Acoustic Sources in Deep-Sea Mining
}

\author{
Valentin Baron, Arthur Finez, Simon Bouley, Florent Fayet, Jérôme I. Mars, Member, IEEE, \\ and Barbara Nicolas, Member, IEEE
}

\begin{abstract}
As the mining of deep-sea natural deposits is becoming cost competitive compared to similar land-based mining, companies have started to dig into the seabeds to collect minerals. However, the acoustic contribution of this activity in the surrounding environment can be significant. To predict the impact of such noise, the starting point is to localize and quantify the sources that create it. In this study, a 3-D prototype acoustic array to perform this localization and quantification is designed, built, and deployed at sea for validation of its localization capacities. The design method performs a two-step study to define the array shape and select the hydrophone arrangement over it, under harsh constraints. Each step relies on two metrics to rank the candidates: the maximum sidelobe level, and the spatial resolution. These are computed on conventional beamforming maps for simulated sources that represent excavation machines on the ground. The shape is first determined to be the one that yields steady maximum sidelobe value levels over frequency. Second, the hydrophone arrangement that achieves the lowest maximum sidelobe level while limiting the spatial resolution is selected. This leads to a tip down conical array with 21 hydrophones, of about $3 \mathrm{~m}$ in height and diameter, and this is manufactured and used during an experimental campaign in the Mediterranean Sea. The experimental localization maps show strong agreement between the estimated source position and its ground truth. A more detailed comparison between simulated and real performances confirms accurate array conception and realization. Thus, this design procedure provides an efficient underwater acoustic array for monitoring deep-sea mining, the localization capacities of which are validated in a real-life setting.
\end{abstract}

Index Terms-Constrained array conception, deep-sea mining, source localization, underwater acoustics.

Valentin Baron is with MicrodB, 69134 Ecully Cedex, France, with the Université de Lyon, INSA-Lyon, Université Claude Bernard Lyon 1, UJM Saint-Etienne, CNRS, Inserm, CREATIS, UMR 5220, U1206, 69100 Lyon, France, and also with the Université Grenoble Alpes, CNRS, Grenoble-INP, GIPSA-Lab, 38000 Grenoble, France (e-mail: valentin.baron@ microdb.fr).

Arthur Finez and Simon Bouley are with MicrodB, 69134 Ecully Cedex,

France (e-mail: arthur.finez@gmail.com; simon.bouley@ microdb.fr).

Florent Fayet is with OSEAN SAS, ZAE La Bayette, 83220 Le Pradet,

France (e-mail: florent.fayet.83@gmail.com).

Jérôme I. Mars is with the Université Grenoble Alpes, CNRS, GrenobleINP, GIPSA-Lab, 38000 Grenoble, France (e-mail: jerome.mars@gipsa-lab. grenoble-inp.fr).

Barbara Nicolas is with the Universite de Lyon, INSA-Lyon, Université Claude Bernard Lyon 1, UJM Saint-Etienne, CNRS, Inserm, CREATIS, UMR 5220, U1206, 69100 Lyon, France (e-mail: barbara.nicolas@creatis.insalyon.fr).

\section{INTRODUCTION}

$\mathbf{T}$ O COPE with the increasing demand for rare minerals, deep-sea natural deposits are becoming attractive again [1]. As a result, companies have started to dig into the seafloor to exploit these, using a range of techniques [2]. Traditionally, these mining sites include excavation machines that dig into the seafloor, with pumps and a riser system to collect the recovered minerals. These need to be linked to a floating production, storage, and offloading unit. All these components obviously radiate noise and contribute to the stress within the nearby environment [3].

Although excavation machines are not acoustically characterized, their spectral shape should be similar to drilling or dredging acoustic sources [4]-[6]. They emit a constant level of $150 \mathrm{~dB}$ between 100 and $500 \mathrm{~Hz}$, and a 20-dB/decade decreasing level from $500 \mathrm{~Hz}$ to $2 \mathrm{kHz}$. The Abysound research project [7] aims to design a prototype for a monitoring system that can localize and quantify these machines on mining sites. This system should deal with slowly moving sources located on the seafloor, and it must provide the same performance in all heading directions. Indeed, with the available deployment techniques, a definite heading cannot be ensured. Moreover, while a position as close as possible to the sources would help to monitor them, security distances must be maintained due to the respective element sizes [8]. These constraints generate the need for a 3-D system with an axisymmetric geometry that can efficiently monitor its environment. This article describes the design steps that are undertaken to achieve such a system, and it includes the validation of its localization capacities through an experimental campaign conducted in the Mediterranean Sea.

An acoustic array is logically chosen to fulfill the requirements, as various examples have shown their strong reliability for underwater localization [9]. However, due to the functional constraints mentioned previously, the array shape has to be carefully chosen. In the literature, only a few acoustic systems have been designed to go in the deep sea, and they all suffer from severe limitations for the context considered here; that of deep-sea mining. Systems that are designed for ambient noise imaging [10], but are not adapted to go in deep waters, have had their extensively detailed designs, which are restricted to 2-D geometries that require heavy positioning efforts. Deep-sea acoustic monitoring stations have also been developed in [11]. However, these have been laid directly on the seafloor and so they cannot monitor the seafloor itself, they must be linked to the coast by electro-optical cables, and their array shape is simplistic, with only four hydrophones. A final example developed to monitor abyssal fauna is linear [12], which prevents a bearing direction of arrival determination. The present study proposes a new design procedure to overcome the limitations of previous acoustic systems, by integrating the constraints at stake into its structure. Moreover, this article deals with a prototype version of the final system, which can fit into a cube of about $3 \mathrm{~m}$ each side for 
transport in an available boat, and where the costs are limited. As a consequence, the design procedure is chosen to follow two steps: first the shape is determined, taking into account a full array size with no cost limitations; and second, the hydrophone arrangement is identified using this shape that is reduced to the prototype size, with a limited number of hydrophones. To stay close to the expected result at full scale, the source emits in a frequency band that is scaled up according to the scale down of the array size during the practical experiment.

To rank the different shapes and hydrophone arrangements, simulated conventional beamforming (CBF) [13] maps are generated for each of them, and their metrics are computed. An efficient array must cover two competing fields: the need to identify a weak source while a powerful source is present within the scanned area, and the need to separate two spatially close sources. This is quantified by two metrics, which are known as the maximum sidelobe level (MSL) and the spatial resolution, respectively, with the spatial resolution evaluated through the "full width at half maximum" (FWHM). As a consequence, an acoustic array that produces the lowest MSL and the highest resolution (i.e., the lowest FWHM) is considered to be best. Nevertheless, such an array cannot be indicated in practice, and the ranking is the result of a compromise between these two metrics, which are represented for all of the tested arrays [14].

The localization of an acoustic source during an experimental campaign in shallow water of the Mediterranean Sea validates both the design and the manufacture of the array. Simulated and practical results are compared in terms of MSL and FWHM, to determine the estimated metrics of the acoustic array, and to fully validate the followed design procedure.

This article is organized as follows. First, the practical constraints that set the boundaries of the possible solutions are detailed in Section II. Then, Section III explains the acoustic model used and the design procedure, and then the array conception choices are developed in Section IV. The measurement campaign is described in Section V, with its results in Section VI. Section VII finally highlights the findings that demonstrate the agreement between the simulated and experimental data.

\section{PRACTICAL CONSTRAintS}

\section{A. Geometric Constraints}

The principal constraint concerns the position of the array in relation to the area under study: the array must not spatially interfere with the machines during their work, particularly as underwater cables represent large obstacles that can damage an array if contact occurs. According to the main recovery technique [8], the array must be positioned to the side of the working zone, to avoid contact with the riser system. This defines a security distance of about $50 \mathrm{~m}$, given the dimensions of the risers involved. The second constraint implies the deployment of the array. An array drops into deep waters by turning on itself. It is therefore heavily expensive to require a definite heading direction for it, once landed on the seafloor. Moreover, the machines are slowly moving on the ground, so they yield various directions for monitoring. A 3-D axisymmetric geometry is the only one that can cope with all of these constraints together. This guarantees the efficiency of the array performance for every heading obtained while allowing monitoring in all directions in space. As a consequence, previous studies that have focused on 2-D geometries cannot be taken as a starting point [10], so a new procedure has to be developed. Finally, the mechanical feasibility of the array structure reduces the possible arrangements: a too complex or random structure cannot be investigated because strong mechanical strength is essential, both to deploy the system at several hundreds of meters in depth, and to avoid the need to compensate for array distortion when processing the signals [15].

Under these constraints, the design procedure aims to define an array that can achieve the following:

1) an MSL of $-8 \mathrm{~dB}$ maximum over the frequency band, which is a classical MSL value that is imposed to be able to relatively easily separate a physical source (represented by its main lobe) from its highest sidelobe;

2) a stable MSL value over frequency, to provide a performance that is independent of the source spectral content;

3) an FWHM limited to the size of the machines that create the expected acoustic sources; e.g., $20 \mathrm{~m}$.

\section{B. Prototype Restrictions}

Due to the ship used for deployment (L'Europe, IFREMER), the array prototype cannot exceed a volume given by a cube of $2.7 \mathrm{~m}$ in height and $2.9 \mathrm{~m}$ in width. This has a direct role on the spatial resolution that can be obtained for this array, as FWHM and array size are proportional [13]. To cope with this limitation, the frequency band of interest $[100 \mathrm{~Hz}, 2 \mathrm{kHz}]$ is increased to allow a reduction in the FWHM, which is also inversely proportional to the frequency [13]. Then, the number of hydrophones is limited to 21 , which restricts the lower bound achievable for the MSL, given that a rough estimate for the MSL is proportional to the number of sensors of the array [16].

Adding together these restrictions, the objectives mentioned previously cannot be reached. Indeed, the hydrophone number limitation prevents low MSL values and impacts on its stability over frequency. However, due to the frequency scaling, the FWHM should remain within the same order of magnitude. Consequently, the design searched for would achieve the lowest and steadiest MSL possible over frequency, trying to stay close to the $-8-\mathrm{dB}$ limit indicated in Section II-A while not drastically degrading the FWHM obtained. The MSL expectations thus lead the choices made in this scheme, where the FWHM has to stay bounded to be under the size of the studied field of view to allow acoustic map interpretations. This can be further justified by the possibility to process localization at a high frequency, due to the wideband signal available in the downsized framework, to obtain a low FWHM, knowing that this issue will not occur any more in the full-size configuration with an array at the correct scale.

\section{METHODS}

\section{A. Acoustic Model}

Usually, in underwater acoustics, the acoustic propagation model is described by plane waves, leading to a free-field propagation vector from an angular direction of arrival $\mathbf{r}$, toward an array hydrophone given by $e^{i k \mathbf{r u}}$, where $\mathrm{k}$ is the wave number, and $\mathbf{u}$ is the vector that describes the hydrophone position compared to the reference hydrophone of the array. However, in the deep-sea mining context studied here, the array is located close to the source, so the curvature of the wavefronts has to be taken into account to correctly describe the delays between the hydrophone signals. Indeed, although the array is in the far-field of the source from an acoustic point of view, given that the acoustic far-field is achieved for a source at a large distance compared to the studied wavelength [17], it is not from a geometric point of view in array processing. Plane wave models can only be used for sources located at large distances compared to $2 L^{2} / \lambda$, where $L$ is the size of the array, and $\lambda$ is the studied wavelength [18]. In our configurations, $L=3 \mathrm{~m}$ and $\lambda_{\text {min }}=c_{0} / f_{\max }=1500 / 7000$, so the source-array distances must be large compared to $84 \mathrm{~m}$, which is not the case. Consequently, free-field spherical wave propagation models the transfer functions between the 


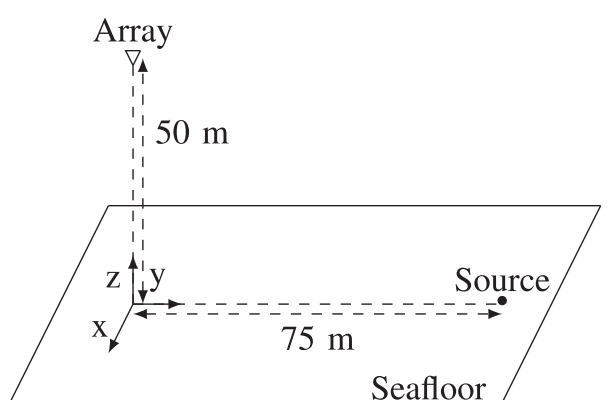

(a)

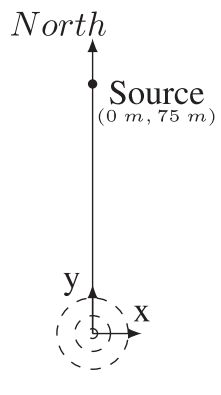

(b)

Fig. 1. Element spatial positions for the simulated configuration. (a) Isometric view: $\nabla$ represents the array located at $(0 \mathrm{~m}, 0 \mathrm{~m}, 50 \mathrm{~m})$, and $\bullet$ represents the source at $(0 \mathrm{~m}, 75 \mathrm{~m}, 0 \mathrm{~m})$. (b) Top view: $\bullet$ represents the source at $(0 \mathrm{~m}, 75 \mathrm{~m})$, and the array is represented by the dashed circles centered on $(0 \mathrm{~m}, 0 \mathrm{~m})$.

array and the different localization points. It follows that localization is performed directly in terms of Cartesian coordinates $(x, y)$ over a grid that spans the plane that corresponds to the seafloor. The propagation vector between one point of this grid and an array hydrophone is denoted by $e^{i k R} / R$, where $R$ is the distance between the hydrophone and the grid point.

\section{B. Design Procedure}

Performance bounds announced in the geometric Section II-A can be expected for a source in the [100 Hz, $2 \mathrm{kHz}]$ band for arrays that are around $10 \mathrm{~m}$ in size and comprise 48 hydrophones. Six axisymmetric shapes that can be actually manufactured are generated and ranked according to the ranking method described in Section III-C. Thereby, the shape identification is achieved for the full-scale configuration, which allows this step to be saved for later studies. Once the shape is chosen, array scaling is applied to go up to the prototype size, and a second design step begins with an array of $2.7 \mathrm{~m}$ in height and $2.9 \mathrm{~m}$ in width. The set of possible hydrophone arrangements is sampled as widely as possible, by allowing reasonable hydrophone positioning, which is both mechanically feasible and relatively uniformly distributed. This even distribution prevents specific performance in a given direction, which must be avoided because of the indefinite heading constraint. The configurations produced are ranked with the same method to indicate the hydrophone arrangement that yields a compromise between lowest MSL and lowest FWHM. Small variations around this local optimum are made to determine whether they provide better performance. To conclude, the behavior of this array is verified within different simulation schemes, to shift from the case used during the design, and to predict its theoretical performance.

\section{Ranking Method}

To sort the different investigated solutions at each step, the MSL and FWHM are both computed from the beampatterns that they each produce. Although a beampattern is traditionally obtained by using a unitary amplitude source in front of the center of an array, the specific configuration of a mining site imposes a steered beampattern to reach good performance with sources located below and to the side of the array. This scheme faithfully represents a possible monitoring system with an array located $50 \mathrm{~m}$ above the ground and to the side of the working zone where the sources that produce noise lie directly on the seafloor. Consequently, as shown in Fig. 1, a unitary source is simulated on the seafloor and $75 \mathrm{~m}$ to the north of the array; so at position $(0 \mathrm{~m}$,

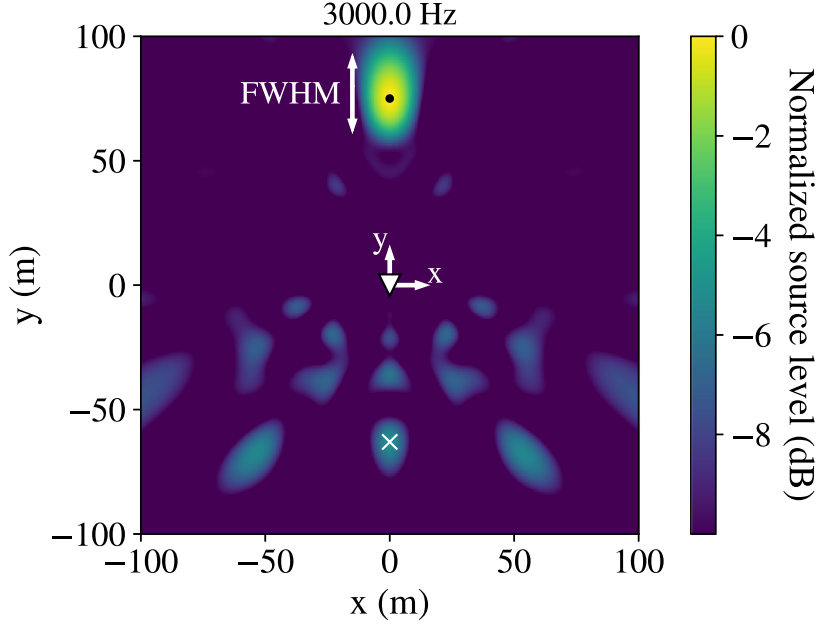

Fig. 2. Example of a CBF map for a source emitting at $3 \mathrm{kHz}$ located at $(0 \mathrm{~m}, 75 \mathrm{~m}, 0 \mathrm{~m})$, represented by $\bullet$. The array is located at $(0 \mathrm{~m}, 0 \mathrm{~m}, 50 \mathrm{~m})$ and its projection on the localization plane is represented by $\nabla$. The white cross indicates the highest sidelobe of the map used to compute the MSL. The FWHM is represented by the double-headed arrow, and is the width at $-3 \mathrm{~dB}$ of the main lobe.

$75 \mathrm{~m}, 0 \mathrm{~m}$ ) within the coordinate system used, as centered on the projection of the array position onto the seafloor. The source is around $90 \mathrm{~m}$ from the array, which justifies the acoustic model chosen. It is important to note that for the rest of this article, the vertical element coordinate (i.e., the $z$-coordinate) is the distance from the seafloor to the element of interest. CBF [13] is performed over the discrete plane centered at $(0 \mathrm{~m}, 0 \mathrm{~m})$ at the source depth (corresponding to the seafloor, $z=0 \mathrm{~m}$ ). This plane will be denoted as the localization plane for the rest of this article. By denoting $\mathbf{g}$ as the steering vector toward a grid point of the localization plane, and $\boldsymbol{\Gamma}$ as the cross-spectral matrix of the measurements, the CBF at the steered grid point steered reads [13]

$$
\mathrm{CBF}_{\mathbf{g}}=20 \log \left(\frac{\mathbf{g}^{*} \boldsymbol{\Gamma} \mathbf{g}}{\operatorname{Tr}(\boldsymbol{\Gamma})\|\mathbf{g}\|^{2}}\right) .
$$

As detailed in [13], (1) outputs a normalized map with a value of $0 \mathrm{~dB}$ at the source position in the presence of a unique source. An example of a CBF map for a source located at $(0 \mathrm{~m}, 75 \mathrm{~m}, 0 \mathrm{~m})$ is given in Fig. 2 This is computed for a $200 \mathrm{~m} \times 200 \mathrm{~m}$ localization plane, with a step size of $0.5 \mathrm{~m}$ and a source at $3 \mathrm{kHz}$. The array is placed at $(0 \mathrm{~m}, 0 \mathrm{~m}$, $50 \mathrm{~m}$ ), and is projected onto the localization plane, as represented by the inverted triangle.

The two previously defined metrics are extracted from the CBF maps as follows.

1) The MSL corresponds to the $\mathrm{dB}$ level of the highest of the sidelobes. In Fig. 2, it is represented by the white cross and is around $-5 \mathrm{~dB}$.

2) The FWHM corresponds to the full width of the main lobe at $3 \mathrm{~dB}$ under the maximum. In Fig. 2, this width is represented by the white double-headed arrow, and it is about $40 \mathrm{~m}$. The major axis length of the ellipse obtained for the main lobe is considered to evaluate this. A high resolution array is then conventionally defined as an array that produces a low FWHM value.

Both of these metrics are then used to rank the different array configurations, with the search for the one that yields low MSL and FWHM. This will ensure separation in both level and space. For each configuration tested, the MSL and the FWHM are computed on the maps produced at different frequencies that correspond to the expected source frequencies. Then, they are averaged over the largest common 


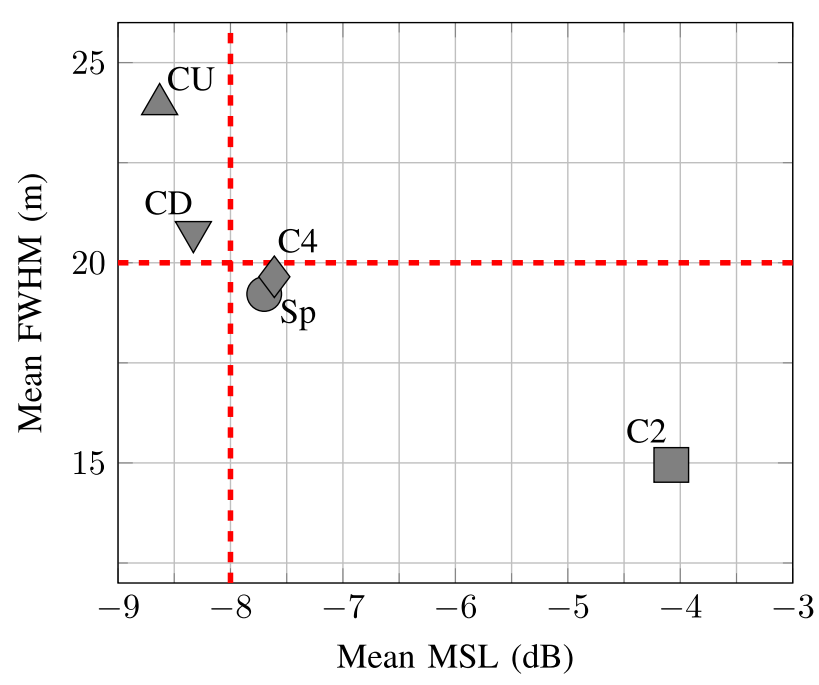

Fig. 3. Array configurations for different geometries within the mean MSL/mean FWHM space. Each marker represents an array shape: conical tip up (CU), conical tip down (CD), cylindrical four circles (C4), spherical (Sp), and cylindrical two circles $(\mathrm{C} 2)$. The frequency bands used for averaging are [160 Hz, $2 \mathrm{kHz}$ ] for the MSL and [180 Hz, $2 \mathrm{kHz}$ ] for the FWHM. - - represents both the MSL and FWHM thresholds given in Section II-A.

frequency band for all of the tested cases, to yield the solution that has the most uniform performance. This is a requirement for the array, as wideband signal recordings are expected as input. This method allows visual and synthetic representation of the performance of various array configurations, by plotting each one in the mean MSL/mean FWHM space, as seen in the array conception in Section IV. Consequently, the array that approaches the nearest to the thresholds defined in Section II can be easily selected from this synthesized view of the array performances.

\section{ARRAY CONCEPTION}

\section{A. Shape Choice}

As detailed in Section III, CBF maps are computed for a source placed at $(0 \mathrm{~m}, 75 \mathrm{~m}, 0 \mathrm{~m})$, and with following six different array shapes:

1) a vertical linear array;

2) two cylindrical arrays (two and four circles of hydrophones);

3) two conical arrays (tip up and tip down);

4) a spherical array.

The 48 hydrophones are positioned as evenly as possible over the chosen shape, and the 10-m size is defined by forcing the two furthest hydrophones of the shape to be $10 \mathrm{~m}$ away from each other.

The MSL and FWHM recovered from the CBF maps are averaged over the frequency bands [ $160 \mathrm{~Hz}, 2 \mathrm{kHz}]$ and $[180 \mathrm{~Hz}, 2 \mathrm{kHz}]$, respectively, with a 20-Hz step. Fig. 3 illustrates the different configurations in the mean MSL/mean FWHM space, where each marker represents a shape.

As the linear array yields a 0-dB MSL because its CBF result gives a circular main lobe centered on itself, it is directly rejected and not represented in Fig. 3. The search for configurations should lie within the bottom left part of the plot, where the MSL and the FWHM are minimal. It can be seen that no configuration reaches this zone. The two shapes of $\mathrm{CU}$ and $\mathrm{C} 2$ in Fig. 3 can be further eliminated due to their great distance from at least one of the thresholds. For the last three shapes possible, a compromise must be found between MSL and FWHM. To perform the selection, the MSL is further studied over frequency by computing its mean and standard deviation for three different frequency bands: 1)
TABLE I

MEANS AND Standard DEVIATIONS OF THE MSL FOR THE THREE CONFIGURATIONS ClOSEST TO THE MSL AND FWHM THRESHOLDS Crossing Over the Three Frequency BAnds, AS [180 Hz, $600 \mathrm{~Hz}]$ (LOW), [600 Hz, $1250 \mathrm{~Hz}$ (MEDIUM), AND [1250 Hz, $2 \mathrm{kHz}](\mathrm{HIGH})$

\begin{tabular}{|c||c|c|c|}
\hline & Low $(d B)$ & $\operatorname{Medium}(d B)$ & High $(d B)$ \\
\hline Sp & $-10.13 \pm 1.70$ & $-7.35 \pm 1.22$ & $-6.48 \pm 0.74$ \\
\hline C4 & $-7.91 \pm 0.02$ & $-7.61 \pm 0.34$ & $-7.40 \pm 0.38$ \\
\hline CD & $-10.31 \pm 0.26$ & $-8.19 \pm 0.80$ & $-7.25 \pm 0.88$ \\
\hline
\end{tabular}

[180 Hz, $600 \mathrm{~Hz}]$, which represents a low frequency band; 2) [600 Hz, $1250 \mathrm{~Hz}]$, which represents a middle frequency band; and 3) $[1250 \mathrm{~Hz}$, $2 \mathrm{kHz}$ ], which represents a high-frequency band. The results are given in Table I.

Considering Table I, the steadiest configuration over all of the frequencies is $\mathrm{C} 4$, with standard deviation bounded below $0.38 \mathrm{~dB}$. However, for the low- and medium-frequency bands, the mean MSL is already higher than the $-8-\mathrm{dB}$ threshold set. For its MSL below $-8 \mathrm{~dB}$ in both the low- and medium-frequency bands and for a standard deviation kept below $0.9 \mathrm{~dB}$ over all of the frequency bands, the $\mathrm{CD}$ configuration must be chosen over that of $\mathrm{Sp}$, which has higher means and standard deviations. By making this choice of $\mathrm{CD}$, it can be seen than the mean FWHM is taken above the 20-m threshold indicated in Section II-A. However, although a low MSL is crucial to obtain readable results in $\mathrm{CBF}$ maps, in the context of underwater mining sites with 20-m-wide sources, the resolution can be relaxed a little. Indeed, these sources should not be too close to one to another (to avoid collisions), which defines the minimum distance between two sources as greater than $20 \mathrm{~m}$. Moreover, the FWHM decreases as the frequency increases, which allows an FWHM of less than $20 \mathrm{~m}$ to be obtained if the processing frequency chosen is high enough.

As a consequence, the CD shape of Fig. 3 is chosen, and Fig. 4 gives its performances for the MSL and FWHM.

Over the entire frequency band, the MSL is not completely stable. It varies between -5.9 and $-16.3 \mathrm{~dB}$, with variations starting from $600 \mathrm{~Hz}$. The 20-m FWHM limit is reached from $1200 \mathrm{~Hz}$, so sources at lower frequencies are difficult to localize accurately with this particular array.

Finally, this shape is acceptable for manufacture due to its good mechanical stability [19], which is essential given the various stresses that can be applied to such arrays [20]. So this can be built without too much complexity, and represents a relevant prototype candidate.

\section{B. Scaling to Prototype}

The 10-m-size conical array is $8.7 \mathrm{~m}$ high, and its widest circle diameter is also $8.7 \mathrm{~m}$. The restrictions that apply to the prototype to build, as detailed in Section II-B, reduce this height to $2.7 \mathrm{~m}$, and the widest circle diameter to $2.9 \mathrm{~m}$. Over this conical shape delimited by these sizes, an infinite number of hydrophone arrangements can be put in place considering that a hydrophone can be positioned anywhere over the cone. Nevertheless, the required mechanical structure drastically reduces the number of possibilities, by requiring discretization of the research space within arrangements that can be actually built. So, the 21 available hydrophones are distributed over the cone within a limited, but reasonable, set of hydrophone positions given by the following:

1) the arrays are formed of $1,2,3,4$, or 5 circles of hydrophones (plus the hydrophone at the tip of the cone);

2) there is a minimum of three hydrophones per circle; 


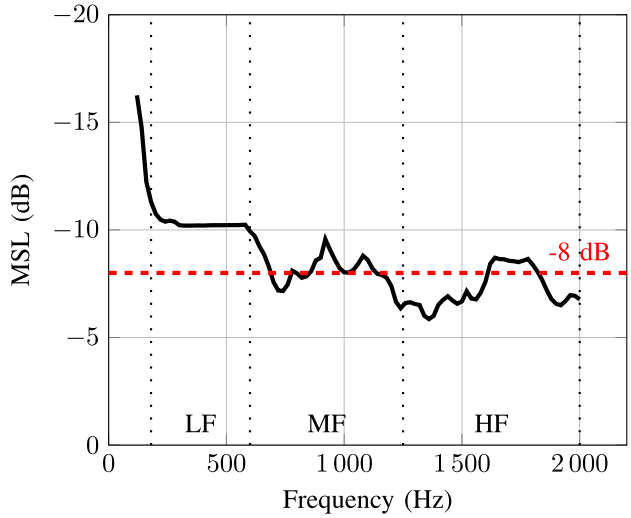

(a)

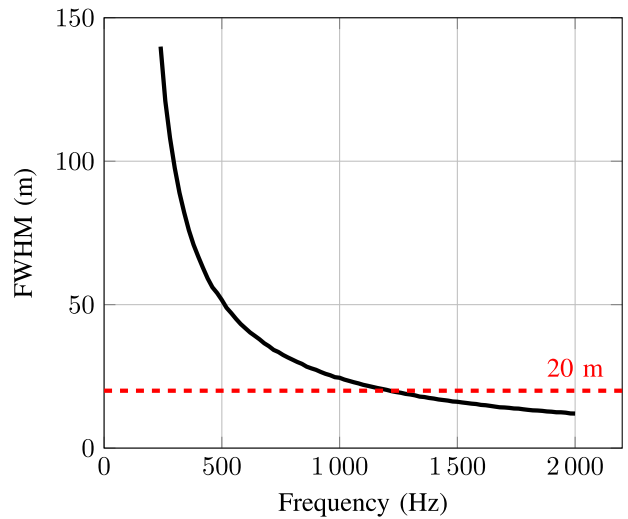

(b)

Fig. 4. (a) MSL and (b) FWHM obtained for the conical tip down (CD) shaped array. - - represents the thresholds given in the MSL and FWHM in Section II-A. The low frequency (LF), medium frequency (MF), and high frequency (HF) bands are defined by the vertical dotted lines in (a).

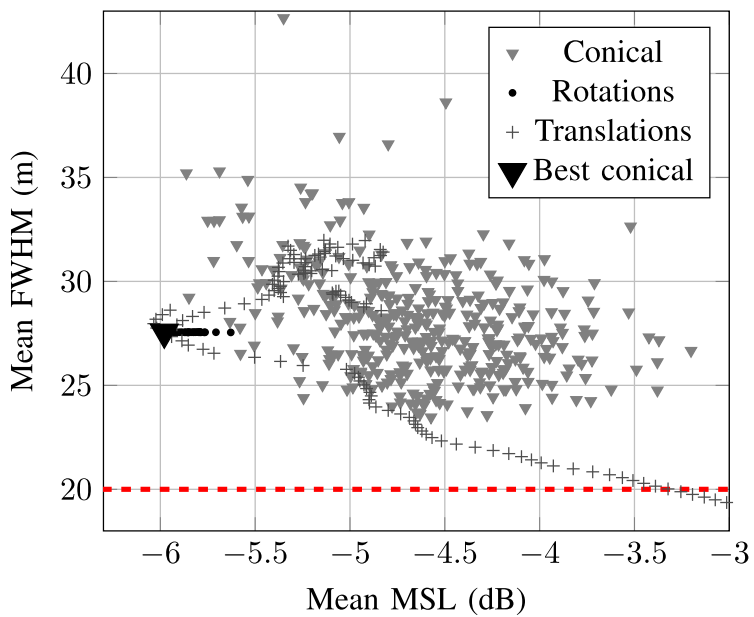

Fig. 5. The 385 different CD configurations in the mean MSL/mean FWHM space. - - represents the threshold given for the FWHM in Section II-A; the MSL threshold cannot be represented because it is too low $(-8 \mathrm{~dB})$ compared to the values obtained $([-6 ;-3] \mathrm{dB})$. Each $\nabla$ represents one of the 385 tested configurations. $\boldsymbol{\nabla}$ represents the configuration that gives the best performance from among these 385 . $\bullet$ gives the performance of a new configuration from the best array with its middle circle rotated by $1^{\circ}$ steps, whereas + gives the performance of a new configuration from the best array with its middle circle translated by 2 -cm steps.

3) the hydrophones have a uniform angular distribution over the circles;

4) the circles are uniformly distributed over the array height.

Taking into account these initial conditions, the number of array configurations to test is 385 . Computing their mean MSL and FWHM over the frequency band [ $800 \mathrm{~Hz} ; 7 \mathrm{kHz}$ ] by steps of $20 \mathrm{~Hz}$ leads to Fig. 5, with these 385 arrays each represented by gray upside-down triangles. The mean MSL is again shown for the $x$-axis, whereas the mean FWHM is shown for the $y$-axis.

These arrays cover MSL values that range from -3 to $-6 \mathrm{~dB}$ and an FWHM from 23 to $43 \mathrm{~m}$, so various performances can be obtained just by moving the hydrophones around within this unique $\mathrm{CD}$ shape. As expected, the mean MSL increases as the hydrophone number is reduced, compared to the larger scale case where 48 hydrophones were available. Conversely, the FWHM stays within the same order of magnitude, with an increase that comes from the higher number of low frequencies used in the average that carries larger FWHM values.

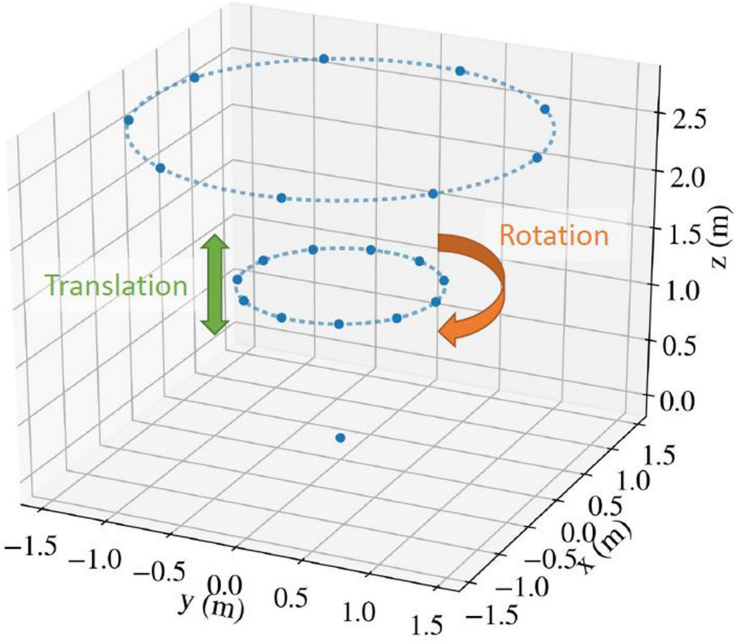

Fig. 6. Illustration of the best array configuration obtained after optimization The dots represent the hydrophone positions. The first hydrophone at the tip of the (inverted) cone is at position $(0 \mathrm{~m}, 0 \mathrm{~m}, 0 \mathrm{~m})$, the first circle is composed of 11 hydrophones at a height $z=1.35 \mathrm{~m}$, with a diameter of $1.45 \mathrm{~m}$, and the second circle is composed of 9 hydrophones at a height $z=2.7 \mathrm{~m}$, with a diameter of $2.9 \mathrm{~m}$.

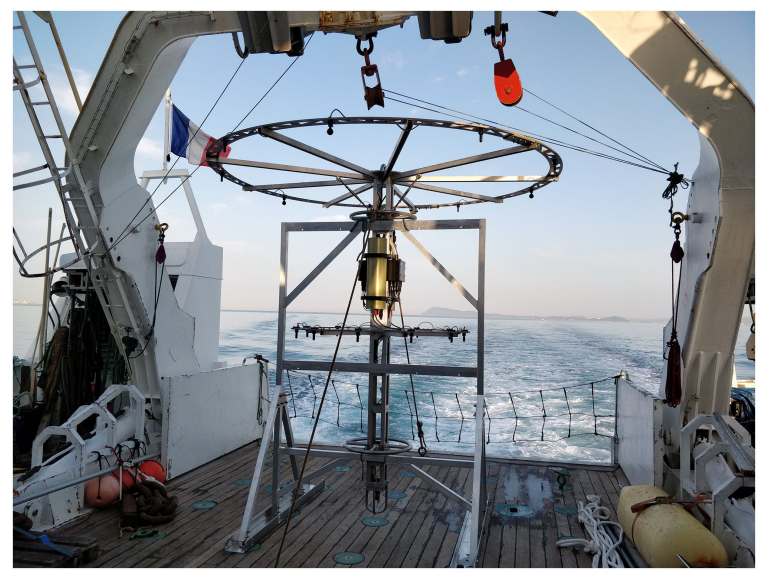

Fig. 7. Final acoustic array obtained. Height: $2.7 \mathrm{~m}$; largest width: $2.9 \mathrm{~m}$. It is composed of 17 HTI-96-MIN and 4 Neptune D/60 hydrophones. (Credits: OSEAN SAS) 


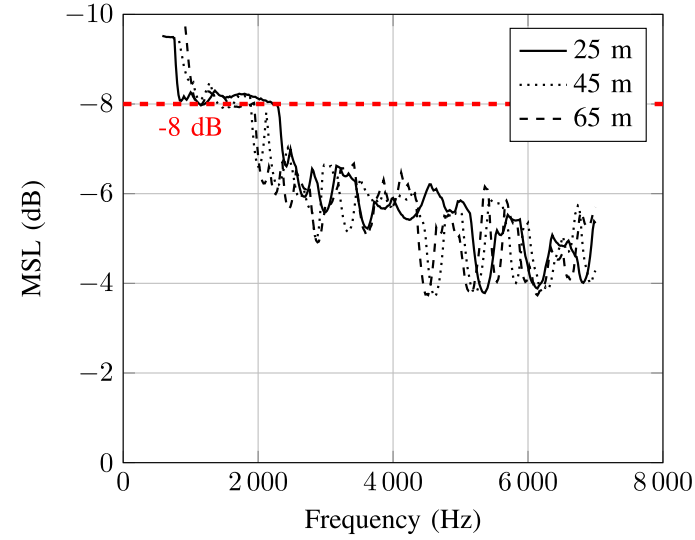

(a)

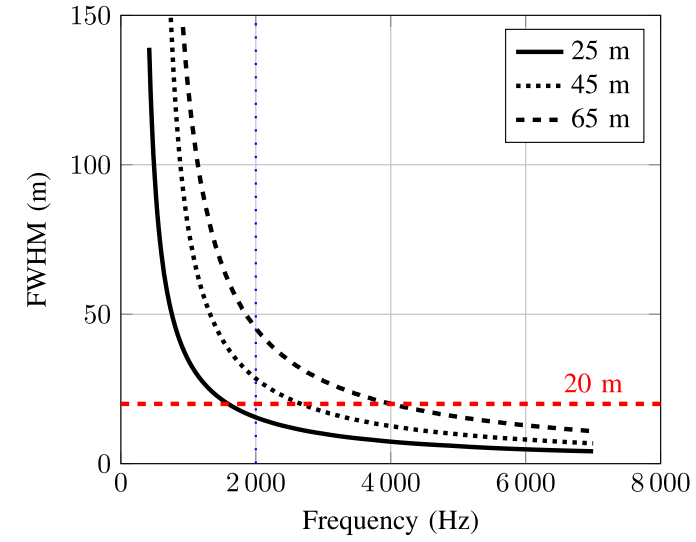

(b)

Fig. 8. (a) MSL and (b) FWHM obtained for 25-, 45-, and 65-m source shifts. The shifts are the distances between the projection of the array vertical axis on the localization plane and the source $y$ coordinates on the plane. - - represents the thresholds given for MSL and FWHM in Section II-A; $\bullet \bullet \bullet$ in (b) represents the 2-kHz frequency, to illustrate the FWHM differences between the cases at this particular frequency: $45 \mathrm{~m}$ for the $65-\mathrm{m}$ shift; $26 \mathrm{~m}$ for the $45-\mathrm{m}$ shift; and $15 \mathrm{~m}$ for the $25-\mathrm{m}$ shift.

From the requirements given in Section II-B, the chosen array arrangement is the only one that achieves $-6 \mathrm{~dB}$ for the mean MSL with a reasonable mean FWHM of $27 \mathrm{~m}$. This is indicated in Fig. 5 by $\boldsymbol{\nabla}$. It is composed of two circles of evenly distributed hydrophones, the first with 11 hydrophones at a height of $1.35 \mathrm{~m}$ and with a diameter of $1.45 \mathrm{~m}$, the second with 9 hydrophones at a height of $2.7 \mathrm{~m}$ and with a diameter of $2.9 \mathrm{~m}$, and with the final unique hydrophone placed at the tip of the cone. This array is illustrated in Fig. 6.

To ensure unbiased optimization in terms of both the angle and the height positions chosen for the circles of hydrophones, these parameters are modified slightly, which provides new configurations that are close to that selected, but that change the performance observed. Fig. 6 illustrates how these modifications can be achieved. On the one hand, the middle hydrophone circle can be turned around the vertical axis with $1^{\circ}$ angular steps, which results in 32 new configurations to test. On the other hand, this middle hydrophone circle can be translated from the bottom $(2 \mathrm{~cm})$ to the top $(2.68 \mathrm{~m})$ of the array height by $2-\mathrm{cm}$ steps, which results in 134 configurations to test. As illustrated by the black dots in Fig. 5, the circle rotations around the vertical axis do not improve either the MSL, which is degraded, or the FWHM, which does not change at all. The translations represented by the gray crosses in Fig. 5 provide much more variation in the performance (which are not all shown, for clarity), but they are again limited to the performance of the original configuration.

Consequently, the former arrangement without rotation or translation of its circles is chosen. The final real array after construction is shown in Fig. 7.

\section{Validation}

The final step to ensure the reliability of the optimization method is to check the performance of the array over the whole frequency band. As the goal is to achieve good performance over all of the frequency band $[350 \mathrm{~Hz}, 7 \mathrm{kHz}]$, particular solutions that would provide excellent results for part of the frequency band, but poor results elsewhere, are not allowed. Moreover, the optimization is conducted for a unique source position, which can be different in the practical deployment. So, validation of the performances with different source positions is required.
A simulation is then conducted with the selected array at $40 \mathrm{~m}$ from the source localization plane. The source is moved over the $y$-axis, from 25 to $65-\mathrm{m}$ by $5-\mathrm{m}$ steps, the $0-\mathrm{m}$ shift being represented by the intersection between the localization plane and the array vertical axis. This simulates different distances between the source and array, as might be the case during the real deployment of the material. The MSL and FWHM obtained are plotted as functions of the frequency in Fig. 8(a) and (b), respectively, for the cases of 25, 45, and $65 \mathrm{~m}$.

These results confirm the comparable performances of the array for various configurations that might occur in the context of the real experiments. For the expected MSL range from -10 to $-4 \mathrm{~dB}$, for increasing frequencies and for all of the source distances, the curves follow the same kind of oscillations that are due to the small number of hydrophones available. The $-8 \mathrm{~dB}$ threshold claimed cannot be respected for high frequencies for the built array, which needs to be kept in mind when analyzing the CBF maps, to avoid misinterpretation. Then, as expected, the FWHM decreases as the source is closer to the array, with values that go from $15 \mathrm{~m}$ for a $25-\mathrm{m}$ shift, to $45 \mathrm{~m}$ for a $65-\mathrm{m}$ shift, at $2 \mathrm{kHz}$

\section{MeAsurement CAmpaign}

To assess the numerical results obtained in the simulation, an experiment was conducted within the Abysound project. This took place in April 2018 in the Cavalaire Bay of Mediterranean Sea, off France, to validate the array construction, but also to test more advanced signal processing methods to localize the sources [21]. The acoustic array was placed $53 \mathrm{~m}$ above the seafloor in a 104-m-deep zone. The acoustic source, placed $24 \mathrm{~m}$ above the seafloor, was a spherical ITC-1001 transducer that emitted $10 \mathrm{~s}$ of sinusoidal signals from 3 to $7 \mathrm{kHz}$, in steps of $1 \mathrm{kHz}$. The geometry of the deployment is detailed in Fig. 9.

As the project source could not be laid on the seafloor without being lost at the end of the experiment, the searched ground truth was the source position in the localization plane parallel to the seabed, at $24 \mathrm{~m}$ above it. This source position was given by a GPS station placed on the boat that carried it. This GPS was shifted $6.5 \mathrm{~m}$ away from the vertical of the source, and it had 2.5-m accuracy in its measurements. As the boat heading could not be determined, this meant that the source position was known with an uncertainty of $9 \mathrm{~m}$. The natural drift of the boat implied 


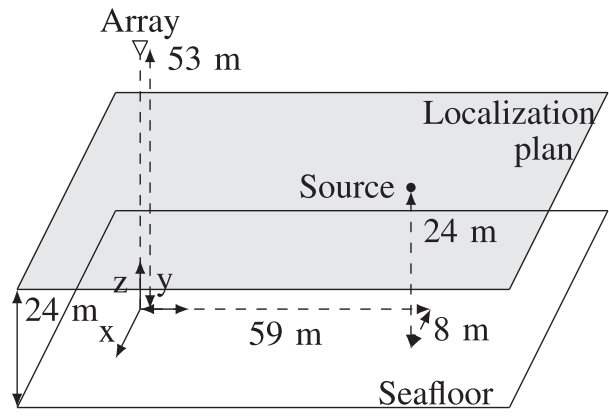

(a)

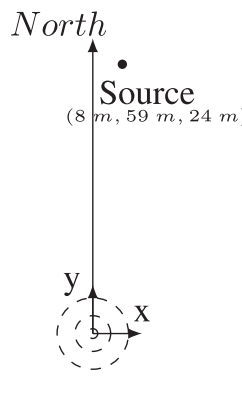

(b)

Fig. 9. Configuration of the shallow water experiment for the $(x, y, z)$ positions. (a) Isometric view: $\nabla$ represents the array located at $(0 \mathrm{~m}, 0 \mathrm{~m}, 53 \mathrm{~m})$, and $\bullet$ the source at $(8 \mathrm{~m}, 59 \mathrm{~m}, 24 \mathrm{~m})$. (b) Top view: $\bullet$ represents the source at $(8 \mathrm{~m}, 59 \mathrm{~m}$, $24 \mathrm{~m})$, and the array is represented by the dashed circles centered on $(0 \mathrm{~m}, 0 \mathrm{~m}$, $53 \mathrm{~m})$.

an angle between the source line and the vertical. Due to the calm weather conditions during the experimental campaign, this estimated angle was reduced to $1^{\circ}$. This produced another uncertainty of about $1.4 \mathrm{~m}$ in the source position on the localization plane. Finally, the source depth was meant to have an accuracy of $1 \mathrm{~m}$, creating a further $1.1 \mathrm{~m}$ of uncertainty in the localization plane. The goal of the acoustic imagery methods was then to find the source within a circle centered on the boat GPS corresponding to $(8 \mathrm{~m}, 59 \mathrm{~m}, 104 \mathrm{~m})$ during the 3-kHz emission and $(9 \mathrm{~m}, 61 \mathrm{~m}, 104 \mathrm{~m})$ during the $7-\mathrm{kHz}$ emission. This circle with a radius of $11.5 \mathrm{~m}$ therefore represents all of the uncertainties detailed before. As the searched plane spanned a square of $200 \mathrm{~m} \times 200 \mathrm{~m}$, this means that the uncertainty area covered $1 \%$ of the scanned zone.

\section{RESULTS}

The CBF computed with the sinusoidal signal at 3 and $7 \mathrm{kHz}$ in Fig. 10(a) and (b), respectively, show the top-view projections of the configuration with the array position represented on the localization plane. The shaded zone corresponds to the uncertainty area, and it is centered on the black dot, which represents the boat GPS. The cross indicates the maximum of the CBF map, and this provides the estimated source position with this method. It can be noted that the normalization is not perfect with real data, with a maximum value of the maps around $-1 \mathrm{~dB}$. This expresses the misalignment between the model and the real propagation that occurs. To cope with this slight decrease, experimental MSL values are computed by comparing the highest sidelobe of the map to the maximum of the map. For every frequency tested, the estimate was localized within the uncertainty area, with a source found for the $3-\mathrm{kHz}$ case at $(3 \mathrm{~m}, 60 \mathrm{~m}, 24 \mathrm{~m})$ and for the $7-\mathrm{kHz}$ case at $(2 \mathrm{~m}, 60 \mathrm{~m}$, $24 \mathrm{~m}$ ), which validates the localization of the source. These results show the gain in FWHM, with values that are reduced at higher frequencies, from 29 to $10 \mathrm{~m}$ at 3 and $7 \mathrm{kHz}$, respectively. Conversely, the MSL increases with higher frequencies, as expected, from -5.1 to $-3.6 \mathrm{~dB}$.

From here, an interesting result is to compare the experimental maps with the simulated ones computed during the design phase. Figs. 10(a) and 2 show the comparison between the experimental and simulated maps. The difference in spreading for the main lobe is due to the shift in the $y$-direction between the simulation source position $(75 \mathrm{~m})$ and the real source position $(59 \mathrm{~m})$. For the sidelobes, the symmetric aspect of the simulation map with a source placed exactly on the $y$-axis explains the differences for the experimental map. Nevertheless, the sidelobe levels are close in both cases, and the maps are very similar.

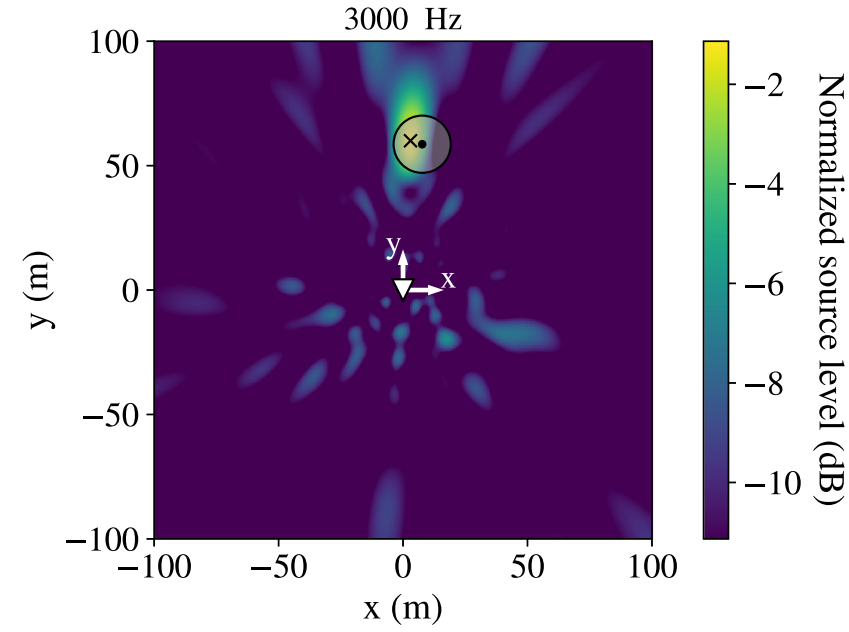

(a)

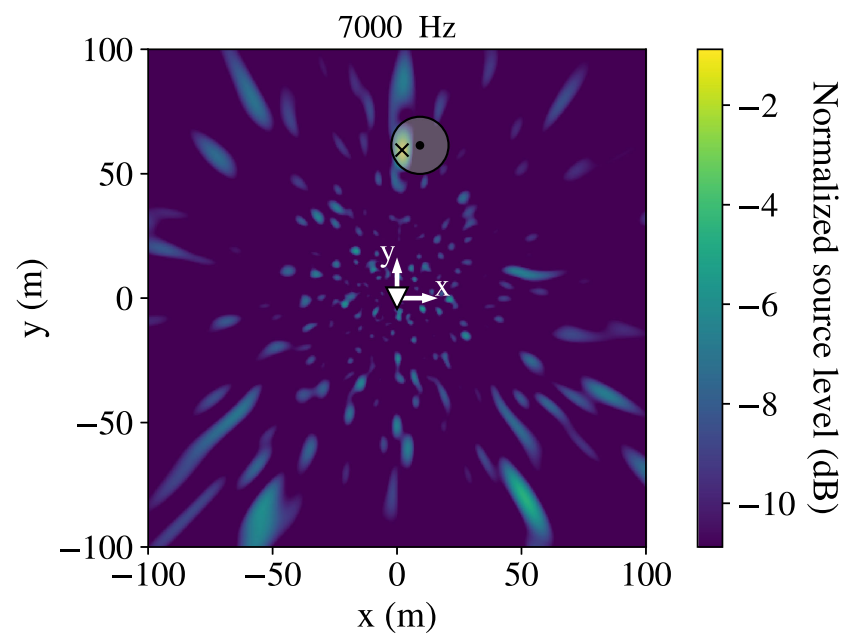

(b)

Fig. 10. CBF maps obtained with the experimental data. (a) At $3 \mathrm{kHz}$, with $\nabla$ as the array located at $(0 \mathrm{~m}, 0 \mathrm{~m}, 53 \mathrm{~m}), \bullet$ as the boat position located at $(8 \mathrm{~m}$, $59 \mathrm{~m}, 104 \mathrm{~m}$ ) (where $104 \mathrm{~m}$ is the water depth), and $\times$ as the source position estimate at $(3 \mathrm{~m}, 60 \mathrm{~m}, 24 \mathrm{~m})$. (b) At $7 \mathrm{kHz}$, with $\nabla$ as the array located at $(0 \mathrm{~m}$, $0 \mathrm{~m}, 53 \mathrm{~m})$, $\bullet$ as the boat position located at $(9 \mathrm{~m}, 61 \mathrm{~m}, 104 \mathrm{~m})$, and $\times$ as the source position estimated at $(2 \mathrm{~m}, 60 \mathrm{~m}, 24 \mathrm{~m})$. The shaded circles of radius $r=11.5 \mathrm{~m}$ represent the uncertainty zone given for the source position in the localization plane.

For a quantified comparison, the MSL and FWHM were determined at each processed frequency for the experimental data. As the real source was located at $59.5 \mathrm{~m}$ away from the array in the $(x, y)$ plane and $29 \mathrm{~m}$ below the array, a new simulation corresponding to this geometric configuration was performed following the procedures of Section IVC. The MSL and FWHM obtained are shown in Fig. 11(a) and (b), respectively, as functions of the frequency. The solid curves represent the simulation results, whereas the gray points give the performance measured with the real data from the experiment.

The results show the experimental MSL points that lie near the simulation curves for each processed frequency. This implies that the experimental data were very close to the expected values in the simulation. Given that an array often shows an increased MSL between the design phase and real use, this result validates the agreement between the simulated and realized arrays. Then, the FWHM points 


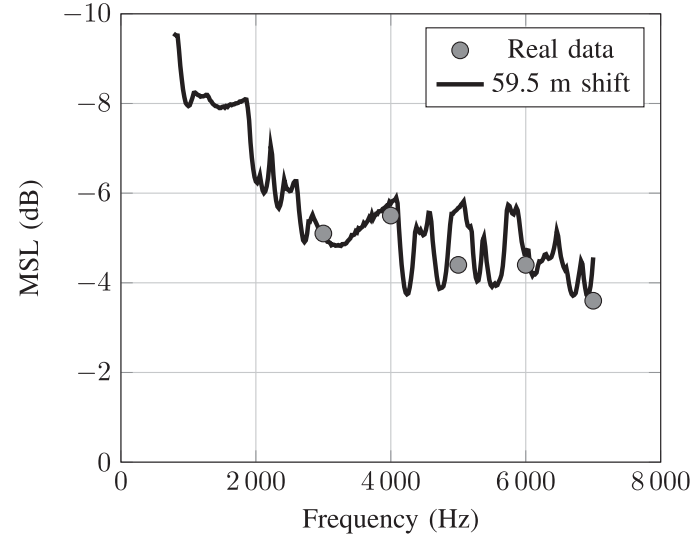

(a)

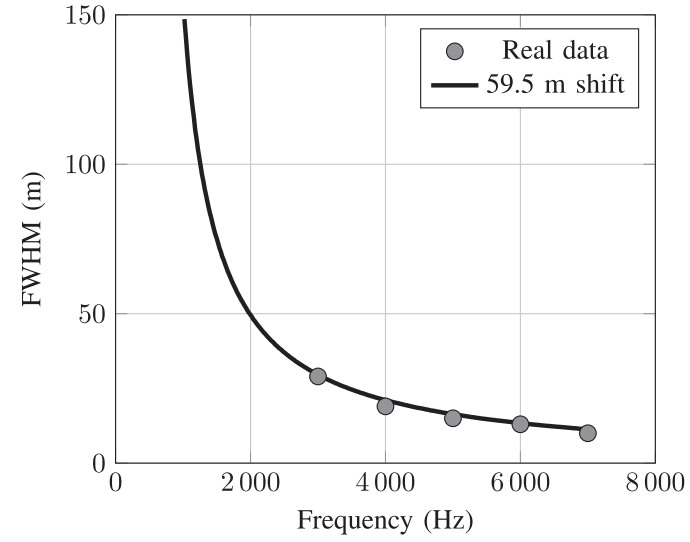

(b)

Fig. 11. Comparison between simulated and experimental performances for the (a) MSL and (b) FWHM. Solid curves represent the simulated array performances for a source located $59.5 \mathrm{~m}$ away from the array in the $(x, y)$ plane, and $29 \mathrm{~m}$ below it, corresponding to the experimental configuration. $\bullet$ are the experimental performances measured from the computed CBF maps for the sinusoidal signals that emit at 3,4,5,6, and $7 \mathrm{kHz}$.

also lie on the theoretical curve, providing again agreement between the experimental and simulated data. This indicates not only that the simulation requirements are fulfilled by the constructed material, but also that the free-field propagation that is assumed by the model is valid in the considered configuration. In other words, even if the source is not laid directly on the seafloor, the reflections on the sediments do not significantly disturb the $\mathrm{CBF}$ estimates.

\section{CONCLUSION}

This article presents the two-step design of an acoustic array dedicated to monitor excavation machines that collect minerals in deep-sea mining. Its shape is first determined according to the specific constraints such that a goal bring, namely, a 3-D axisymmetric geometry. Once the shape is decided and reduced to respect the prototype restrictions, the hydrophone arrangement that produces the lowest sidelobes in simulation maps is selected. This is more extensively validated against various configurations that can occur in practice, and manufactured to produce a 2.7-m-high conical shape array with tip down of 21 hydrophones, with its widest circle of hydrophones of 2.9-m diameter. This was deployed in a 100-m-deep zone during an experimental campaign conducted in the Mediterranean Sea, to validate its localization abilities based on a transducer positioned $24 \mathrm{~m}$ above the seafloor that emitted sinusoidal signals. All the emitted signals are found within an uncertainty boundary drawn from the position device inaccuracies. Thus, the array design and manufacturing fulfill localization goals aimed for with this monitoring system. The chosen methods also show robustness to model errors, with a source correctly localized where reflections on the ground occur. To progress toward a complete monitoring system, the quantification capacities must be assessed, and if possible at greater depth, to answer all the challenges that such a system might be exposed to.

\section{ACKNOWLEDGMENT}

The authors would like to thank all the partners of the Abysound project: Naval Group, OSEAN SAS, MicrodB, Semantic TS, IFREMER, Gipsa-Lab, Laboratoire de Mécanique et d'Acoustique, and Laboratoire des Sciences de l'Information et des Systèmes. This work was performed within the framework of the LABEX CeLyA
(ANR-10-LABX-0060) of Université de Lyon, within the program Investissements d'Avenir (ANR-16-IDEX-0005) operated by the French National Research Agency (ANR).

\section{REFERENCES}

[1] G. Glasby, "Lessons learned from deep-sea mining," Sci., vol. 289, no. 5479, pp. 551-553, Jul. 2000.

[2] G. Spagnoli, S. A. Miedema, C. Herrmann, J. Rongau, L. Weixler, and J. Denegre, "Preliminary design of a trench cutter system for deep-sea mining applications under hyperbaric conditions," IEEE J. Ocean. Eng., vol. 41, no. 4, pp. 930-943, Oct. 2016.

[3] S. Gollner et al., "Resilience of benthic deep-sea fauna to mining activities," Mar. Environ. Res., vol. 129, pp. 76-101, Aug. 2017.

[4] K. Reine, D. Clarke, and C. Dickerson, "Characterization of underwater sounds produced by hydraulic and mechanical dredging operations," $J$. Acoust. Soc. Amer., vol. 135, no. 6, pp. 3280-3294, Jun. 2014.

[5] J. Nedwell and D. Howell, "A review of offshore windfarm related underwater noise sources," Collaborative Offshore Wind Res. Environ., London, U.K., Report 544 R 0308, Oct. 2004.

[6] M. Broudic et al., "Underwater noise emission from the NOAH's drilling operation at the NaREC site, Blyth, UK," in Proc. 2nd Int. Conf. Environ. Interact. Mar. Renewable Energy Technol., May 2014, Paper EIMR2014992.

[7] R. Bassila et al., "Abysound, noise impact measurement of deep sea mining production tools", in Proc. MTS/IEEE OCEANS Conf., Jun. 2019, pp. 1-9.

[8] C. Yu and Y. Cheng, "System and method of utilizing monitoring data to enhance seafloor sulfide production for deep water mining system," USA Patent US 7784201 B2, Aug. 2010.

[9] K. Foote, "Underwater acoustic technology: Review of some recent developments," in Proc. MTS/IEEE OCEANS Conf., Sep. 2008, pp. 1-6.

[10] V. Pallayil, M. Chitre, S. Kuselan, A. Raichur, M. Ignatius, and J. Potter, "Development of a second-generation underwater acoustic ambient noise imaging camera," IEEE J. Ocean. Eng., vol. 41, no. 1, pp. 175-189, Jan. 2016.

[11] G. Riccobene, "Long-term measurements of acoustic background noise in very deep sea," Nucl. Instrum. Methods Phys. Res. Sec. A, Accelerators, Spectrometers, Detectors Associated Equip., vol. 604, no. 1, Supplement, pp. S149-S157, Jun. 2009.

[12] K. Richter, J. Bennett, and K. Smith, "Bottom-moored acoustic array to monitor density and vertical movement of deep-sea benthopelagic animals," IEEE J. Ocean. Eng., vol. OE-10, no. 1, pp. 32-37, Jan. 1985.

[13] G. Elias, "Aeroacoustic and active noise control - Experimental techniques for source location," Lecture Series, Von Karman Inst. Fluid Dyn., SintGenesius-Rode, Belgium, Sep. 1997.

[14] E. Sarradj, "A generic approach to synthesize optimal array microphone arrangements," in Proc. Berlin Beamforming Conf., 2016, pp. 1-12. 
[15] B. Ferguson, "Remedying the effects of array shape distortion on the spatial filtering of acoustic data from a line array of hydrophones," IEEE J. Ocean. Eng., vol. 18, no. 4, pp. 565-571, Oct. 1993.

[16] S. Holm, A. Austeng, K. Iranpour, and J.-F. Hopperstad, "Sparse sampling in array processing," in Non Uniform Sampling. New York, NY, USA: Springer, 2001, pp. 787-833.

[17] P. Morse and K. Ingard, Theoretical Acoustics. Princeton, NJ, USA: Princeton Univ. Press, Jan. 1987, ch. 7.1.

[18] R. Kennedy, T. Abhayapala, and D. Ward, "Broadband nearfield beamforming using a radial beampattern transformation," IEEE Trans. Signal Process., vol. 46, no. 8, pp. 2147-2156, Aug. 1998.

[19] F. Fayet, V. Baron, B. Nicolas, J. Jaumont, A. Finez, and J. Mars, "Conception of an underwater prototype array: from simulation design to mechanical assessment," in Proc. MTS/IEEE OCEANS Conf., Jun. 2019, pp. $1-9$.

[20] T. Delmer, T. Stephens, and J. Tremills, "Numerical simulation of cabletowed acoustic arrays," Ocean Eng., vol. 15, no. 6, pp. 511-548, Jan. 1988.

[21] G. Pham, V. Baron, A. Finez, J. Mars, and B. Nicolas, "High resolution source localization in underwater acoustics for deep sea mining monitoring," in Proc. MTS/IEEE OCEANS Conf., Jun. 2019, pp. 1-7.

Valentin Baron was born in 1993 in France. He received the master's degree in signal processing from the Institut National Polytechnique de Grenoble (part of the University Grenoble Alpes), Grenoble, France, in 2017.

He is currently a CIFRE Ph.D. student (Ph.D. within a company) with MicrodB Ecully, France, co-supervised by the Centre de Recherche en Acquisition et Traitement de l'Image pour la Santé laboratory. His current research interests include array processing and machine learning applied to acoustic environments, with applications such as underwater acoustic localization and quantification, and drone identification through acoustic array measurements.

Arthur Finez was born in 1984 in France. He received the Ph.D. degree (dissertation in experimental aeroacoustics) from the Laboratoire de Mécanique des Fluides et d'Acoustiques, Ecully, France, in 2012.

He has worked for seven years at MicrodB Ecully, France, as an Engineer specialized in microphone array techniques with automotive and aeronautic applications. He is currently an Engineer with Engie Green, Montpellier, France, working in wind turbine acoustics.

Simon Bouley graduated from the École Nationale Supérieure d'Ingénieurs du Mans, Le Mans, France, in 2012. He received the M.S. degree in acoustics from the Université du Maine, Le Mans, France, in 2012, and the Ph.D. degree in aeroacoustics from the École Centrale de Lyon, Lyon, France, in 2017.

From 2017 to 2018, he held a postdoctoral position in acoustic imagery field with the Institut National des Sciences Appliquées, Lyon, France. He is currently an Acoustic Project Manager and Industrial Researcher with MicrodB, Écully, France. His research is mainly related to the enhancement of inverse microphone-array techniques in aeroacoustics by the use of advanced signal processing.
Florent Fayet was born in 1986 in France. He received the master's degree in physics and engineering sciences from the University of Toulon, Toulon, France, in 2010.

He was a Study Engineer and Project Manager in acoustic and signal processing with OSEAN SAS, Le Pradet, France, from 2011 to 2020 . He is currently an Acoustic Engineer with the DGA.

Jérôme I. Mars (Member, IEEE) was born in 1962. He received the master's degree in mechanics and geophysics from the University Joseph Fourier (currently University Grenoble Alpes), Grenoble, France, in 1986, and the Ph.D. degree in signal processing from the Institut National Polytechnique de Grenoble, Grenoble, France, in 1988.

He was with the Centre des Phénomènes Aléatoires et Geophysiques de Grenoble (CEPHAG) between 1989 and 1992; with the Materials Sciences and Mineral Engineering (MSME) Department, University of California, Berkeley (between 1992 and 1995) and the Laboratory of Images and Signal between 1995 and 2007. He is currently a Professor (C-Ex) with Grenoble-INP (Grenoble Institute of Technology), Grenoble, France. He is a Head of the Grenoble Image Parole Signal Automatic Laboratory. His research interests include statistical signal processing and source separation with antennas. In particular his latest research works mostly focus on wave propagation (underwater acoustics, geosciences, and tomography). With more than 90 peer-reviewed publications and 250 international conferences, he is involved in the supervision of more than $35 \mathrm{Ph}$.D. students relating to his research interest.

Barbara Nicolas (Member, IEEE) received the graduate degree from the École Nationale Supérieure des Ingénieurs Electriciens de Grenoble, Grenoble, France, in 2001, and the M.S. and Ph.D. degrees in signal processing from Grenoble-INP, Grenoble, France, in 2001 and 2004, respectively.

From 2006 to 2014, she was a CNRS Researcher with GIPSA-Lab, Image Signal Department, Grenoble, France. In 2008, she was a Visiting Scientist with the Signal Processing Laboratory, Faro, Portugal. Since 2014, she has been a CNRS Researcher with Creatis Lab., Lyon, France. In 2020, she was a Visiting Scientist at Herriot Watt University, Edinburgh, U.K. Her research interests include signal processing in underwater acoustics and medical ultrasound imaging, including array processing, acoustic tomography, time-frequency representations, and underwater source detection and localization. 